\title{
Effect of Ascorbic Acid Treatment on Fruit Quality of Kiwifruit during Storage
}

\author{
Yuan $X Z^{1, a}$, Xia $H^{1,2, b}$, Wang $Y^{1, c}$, Liang $D^{1,2, d^{*}}$ \\ 1 College of Horticulture, Sichuan Agricultural University, Chengdu 611130, China; \\ 2 Institute of Pomology and Olericulture, Sichuan Agricultural University, Chengdu 611130, China \\ a1723065255@qq.com, b381683619@qq.com, c1336489390@qq.com, d756141959@qq.com \\ \#Co-first author, *Corresponding author
}

\section{Keywords: Kiwi; Ascorbic acid; Storage after harvest}

Abstract. The fruit of kiwi is rich in nutrients and is deeply loved by people. However, the storage period is not long, and the shelf period is short and easy to be soft. In this experiment, the effects of ascorbic acid on the post-harvest storage of kiwifruit were studied by measuring the quality of the fruit with a solution of $100 \mathrm{mmol} \cdot \mathrm{L}^{-1}$ ascorbic acid based on the fruit of 'Jinshi No. 1'. The results showed that ascorbic acid treatment reduced fruit quality in 7-14 days compared with control(CK). Total flavonoids, and total flavanols showed a smaller decrease, and the antioxidant capacity showed an upward trend or a smaller decrease. Therefore, it is considered that anti-cyclic acid treatment can improve fruit quality and antioxidant properties, and it is an alternative method for storage and preservation of kiwifruit after harvest.

\section{Introduction}

Kiwifruit is a deciduous vine of the genus Actinidia in the kiwifruit family. Its soft texture, sweet and sour juice, vitamin C content is as high as $100-420 \mathrm{mg} \cdot 100 \mathrm{~g}^{-1}$, so it is loved by many people. At the same time kiwifruit contains many tannin pectin, soluble sugar, protein, and a variety of amino acids and trace elements required by humans. According to the test of the Food Research Center of Rutgers University in the United States, kiwifruit is the most abundant and most comprehensive fruit in various fruits ${ }^{[1]}$.

Kiwifruit is a kind of respiration-changing fruit, it has obvious physiological after-ripening process, and the post-harvest fruit is apt to soft rot. Therefore, the kiwifruit has the disadvantage of storage failure ${ }^{[2]}$. Kiwi fruit ripening and senescence are complex physiological and biochemical processes. This process includes the synthesis of ethylene, the degradation of cell walls, the softening of fruits, the hydrolysis of starch and the accumulation of sugar, the change of pulp color, and the formation of aromatic substances ${ }^{[3-5]}$.

Ascorbic acid is an important antioxidant substance in plants. It is a water-soluble vitamin and has important physiological control functions in the plant's own growth and development. It can regulate plant growth, induce flowering, and delay the aging process of fruits and vegetables. Absence of AsA in plants will reduce the resistance of plants in the adverse environment. ASA can not only participate in the elimination of hydrogen peroxide involved in ascorbate peroxidase intervention on the surface of chloroplast thylakoids as a reducing agent; it can also directly remove reactive oxygen species as an antioxidant. It plays an important role in protecting cell membrane structure and reducing membrane lipid peroxidation. Many studies have shown that the appropriate concentration of AsA treatment can extend the shelf life of horticultural products such as crystal pears, apricots, grapes, litchi, and cherry tomatoes ${ }^{[6]}$.

\section{Materials and Methods}

Materials. 'Hongyang' kiwifruits used in this study were harvested from Kiwifruit Resource Orchard in Shifang $\left(104^{\circ} 16^{\prime} \mathrm{N}, 31^{\circ} 13^{\prime} \mathrm{E}\right)$, Chengdu, China. During the fruit harvest period, the fruit is harvested when the fruit's physiological maturity reaches (TSS $\geq 6-8$ ), and then transported back 
to the laboratory quickly after harvest. Select fresh fruit with uniform size, consistent maturity, no pests and diseases, and no mechanical damage as test materials.

Preparation of kiwifruit extracts. The selected kiwi fruit was immersed in a $5 \%$ hypochlorous acid solution for sterilization. After 10 minutes, they were randomly divided into two groups of 30 fruits. The first group was immersed in distilled water for 30 minutes as a control. The second group was soaked in $100 \mathrm{mmol} \cdot \mathrm{L}-1$ ascorbic acid for $30 \mathrm{~min}$. Put the soaked fruits in a cool and ventilated place to dry, then put them in a refrigerator at $4{ }^{\circ} \mathrm{C}$ for storage. Samples were taken 5 times, once every 7 days, and 6 kiwifruits were randomly selected each time. The removed fruits were diced and stored at $-80{ }^{\circ} \mathrm{C}$ for other indicators.

Determination of indicators. Determination of total phenols, total flavonoids, and total flavanols is based on the method of Wang Xiaoqian ${ }^{[7]}$; The determination of free radical scavenging capacity (DPPH), ABTS + radical scavenging ability, and iron ion reduction capacity (FRAP) are based on Du Guorong's method ${ }^{[12-16]}$. The above indicators were repeatedly measured three times or more, and the average value was taken as the final data of each treatment.

Data analysis. Excel 2007 was used for data processing and graphing. Analysis of variance was performed by the SPSS software (SPSS, Inc., Chicago, IL, USA).

\section{Results and Analysis}

Effect of Ascorbic Acid Treatment on Phenolic Compounds in Kiwi Fruit. The phenolic compounds in plants can eliminate free radicals caused by plants. Therefore, phenols play an important role in the resistance physiology of plants. During the treatment of this experiment, the application of ASA significantly increased the content of phenols (Figures 1A, B, C). The content of TPC in the experimental group changed little, but it was always higher than the CK group (Fig. 1A); The experimental group TFC reached a maximum at day 14, which was an increase of $448.3 \%$ over day 0 and began to decline after 14 days (Fig. 1A). The TFAC content reached a maximum on day 7 and increased $444.3 \%$ on day 0 . It began to decrease after 7 days. The contents of TPC, TFC and TFAC showed a trend of increasing first and then decreasing in the experimental group.
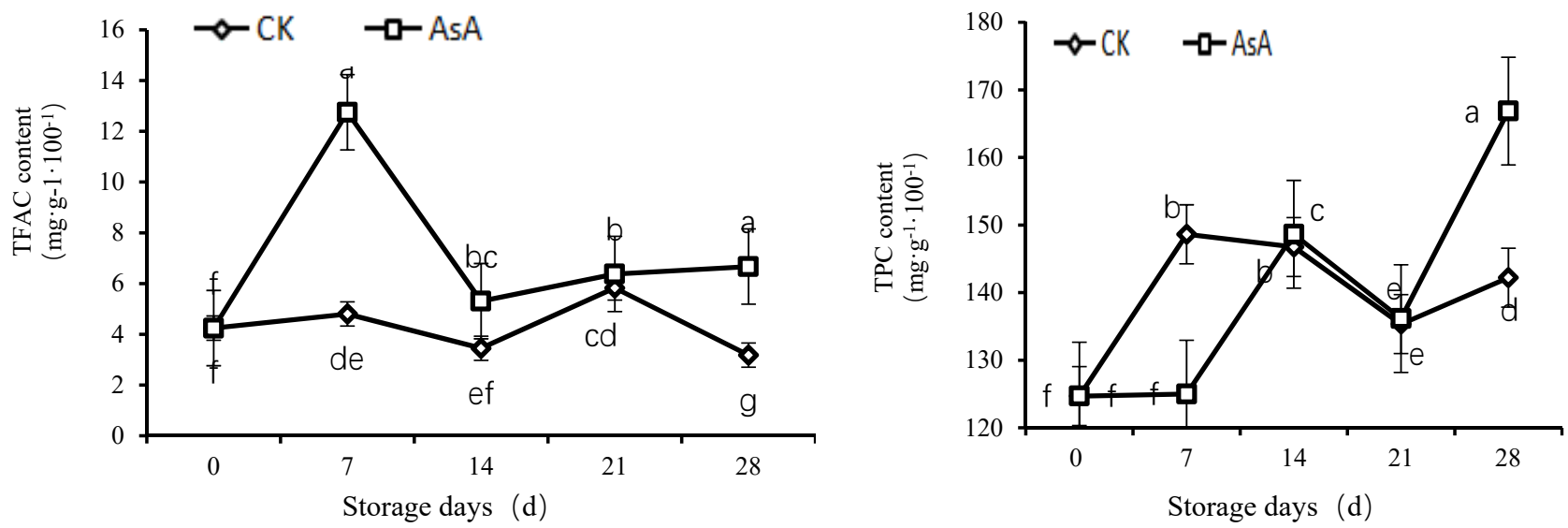


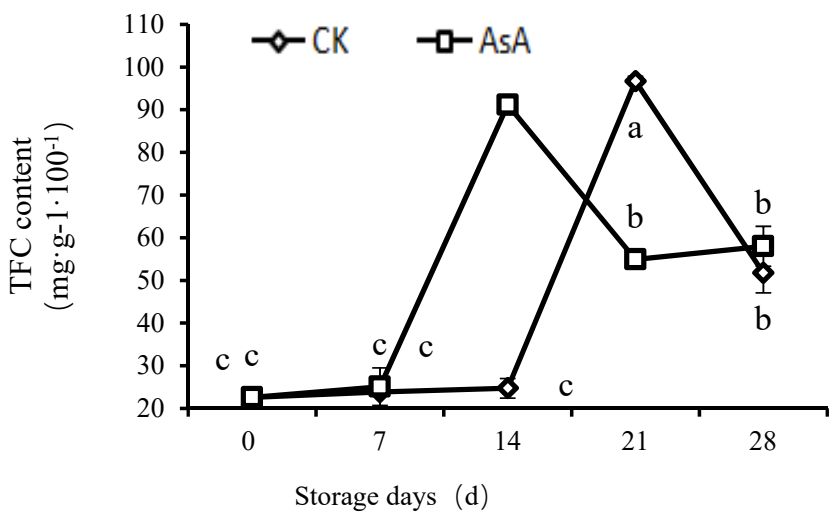

Fig. 1 Effects of ASA application on contents of TPC, TFC and TFAC of kiwifruit leaves.

Effect of Ascorbic Acid Treatment on Antioxidant Capacity of Kiwi Fruit. For the antioxidant capacity of fruit after AsA treatment, the experimental group had a significant effect on FRAP and ABTS except that the change of DPPH was not significant. The changes in the total ABTS content in the two groups are approximately the same. In the first 14 days, the data of ABTS and FRAP in the control group and the treatment group showed a tendency to increase, but the treatment group was lower than the control group. From the 14th to the 21st day, the data of ABTS and FRAP in the control group began to decline, while the data in the treatment group did not change significantly, but its data was still higher than the control group. From the 21 st day to the 28 th day, the FRAP content increased again. On the 28th day, the content of the treatment group was significantly higher than that of the control group.
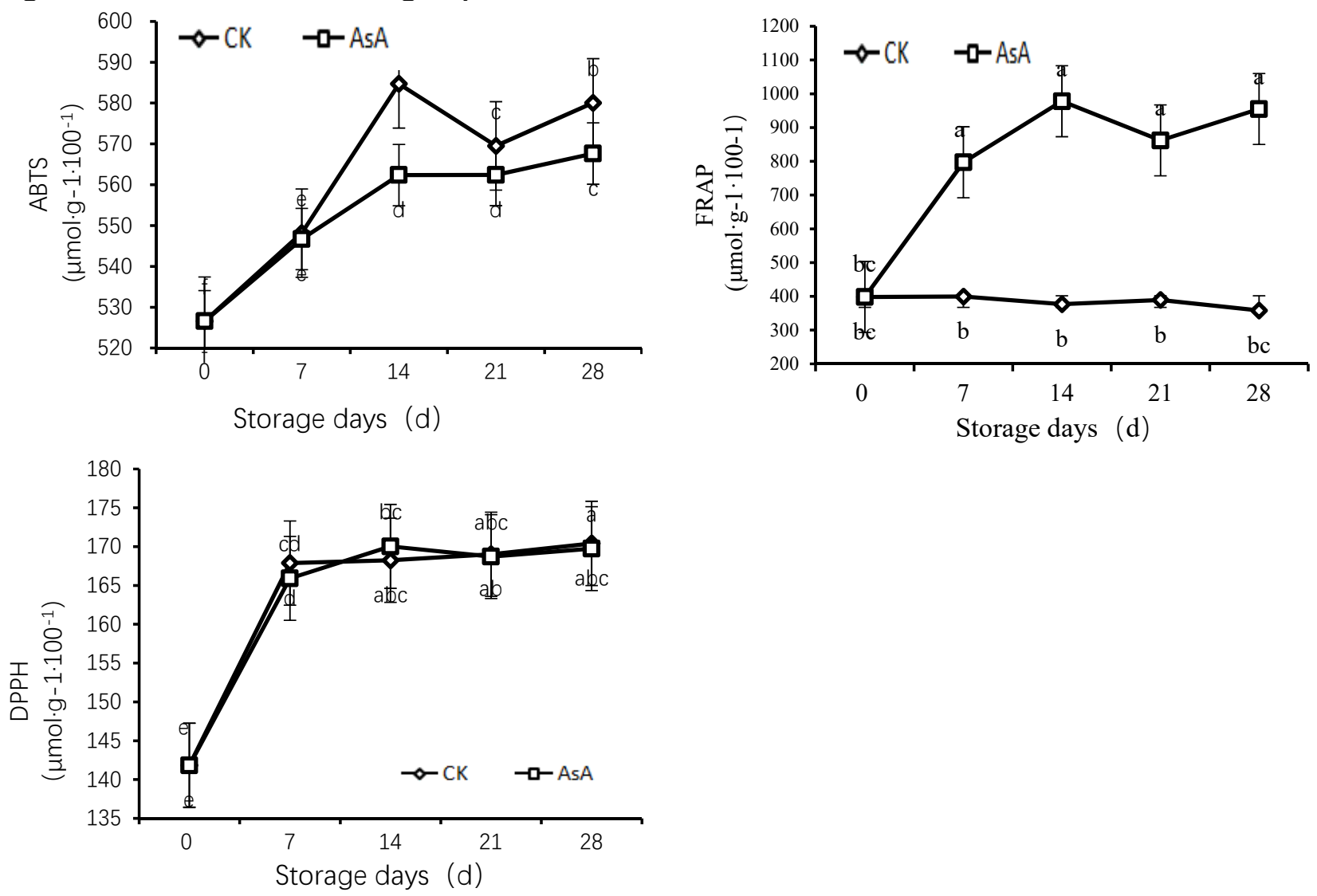

Fig. 2 Effects of ASA application on FRAP, ABTS and DPPH of kiwifruit leaves.

\section{Conclusions}

After AsA treatment, the contents of total flavonoids, total flavanols and total phenols in fruits increased or slowed the rate of decline down. Among the indicators of antioxidant capacity, only the change of DPPH was not significant, while the effect of ASA treatment on FRAP and ABTS was 
more significant. From this, it can be concluded that treatment of $100 \mathrm{mmol} \cdot \mathrm{L}^{-1}$ ascorbic acid at 7-14 d slows fruit quality decline. Total phenols, total flavones, and total flavanols show increasing trends or slowed the rate of decline down. Antioxidant ability is improved. Therefore, it can be considered that treatment with ascorbic acid can improve fruit quality and enhance antioxidant activity. This treatment is an effective method for post-harvest storage of kiwifruit.

\section{Acknowledgements}

This work was financially supported by the Sichuan Technical Supports Foundation (2016NZ0105) and Training Programs of Innovation for Undergraduates in Sichuan (201610626057).

\section{References}

[1] Zhao S, Wang M, Yang G, et al. Assessment of Fruit Quality of Twelve Kiwifruit Varieties Introduced and Planted in China[J]. Research of Agricultural Modernization, 2002, 23(6):455-457.

[2] Wang S, Yang J, Duan C, et al. Advance of Research on Mature Physiological and Preservation Technology of Kiwifruit[J]. Chinese Agricultural Science Bulletin, 2013.

[3] Wang S, Yang J, Duan C, et al. Advance of Research on Mature Physiological and Preservation Technology of Kiwifruit[J]. Chinese Agricultural Science Bulletin, 2013.

[4] Chen J, Zeng R, Li P. Advance of Research on Postharvest Physiology of Kiwifruit and Its Storage Technology[J]. Acta Agriculturae Universitis Jiangxiensis, 2002, 24(4):477-483.

[5] Zhang H, Ma C, Peng X, et al. Advances of Research on Postharvest Physiology and Disease of Hongyang Kiwifruit[J]. Guangdong Chemical Industry, 2017.

[6] Cho J S, Jeong M C, Moon K D. Effects of Ultrasound and Ascorbic acid Cotreatment on Browning of Fresh-cut 'Tsugaru' Apples[J]. Maternal \& Child Nutrition, 2012, 19(3):323-327.

[7] Chun-Hua M A, Zhang M, Ming-Jun L I, et al. Influence of Exogenous Ascorbic Acid on Postharvest Ripening and AsA-GSH Cycles in Apple Fruit[J]. Journal of Yunnan Agricultural University, 2012.

[8] Zhang J Q, Zhang J G, Ai-Li F U, et al. Effect of Exogenous Antioxidants on the Enzymatic Activities in Fruit Peel Tissues under High-temperature Stress[J]. Acta Agriculturae Boreali-sinica, 2005.

[9] Mo Y, Zheng J, Li W, et al. Effects of ascorbic acid and glutathione treatments on litchi fruits during post-harvest storage[J]. Transactions of the Chinese Society of Agricultural Engineering, 2010, 26(3):363-368.

[10] Liu K, Jing G, Yuan C, et al. Effect of Ascorbic Acid Treatment on Physiology and Antioxidants of Cherry Tomato Fruits during Storage[J]. Chinese Journal of Tropical Crops, 2012. 